\title{
Dispareunia en mujeres después de parto vaginal. Prevalencia en dos clínicas de Armenia, Colombia, 2012-2017
}

\section{Dyspareunia in women after vaginal delivery. Prevalence in two clinics in Armenia, Colombia, 2012-2017}

\author{
Franklin J. Espitia-De La Hoz (iD ${ }^{1}$
}

1. Universidad Militar Nueva Granada. Bogotá, Colombia. Correo: espitiafranklin@hotmail.com - https://orcid.org/0000-0002-4581-9680

Tipología: Artículo de investigación científica y tecnológica

Para citar este artículo: Espitia-De La Hoz FJ. Dispareunia en mujeres después de parto vaginal. Prevalencia en dos clínicas de Armenia, Colombia, 2012-2017. Duazary. 2021 enero; 18(1): 32-44. Doi: https://doi.org/10.21676/2389783X.3887

Recibido en febrero 20 de 2020

Aceptado en octubre 18 de 2020

Publicado en línea en enero 07 de 2021

Palabras

clave:

dispareunia;

prevalencia;

parto normal;

mujeres;

episiotomía.

\section{RESUMEN}

Se realizó un estudio de corte transversal que tuvo como objetivo determinar la prevalencia de la dispareunia en mujeres con parto vaginal, evaluar posibles factores de riesgo y la asociación con la episiotomía. Ingresaron mujeres atendidas por parto vaginal entre 2012 y 2017 en dos clínicas de alta complejidad en Armenia (Colombia). Se utilizó el cuestionario Índice de Función Sexual Femenina (IFFS6) para evaluar la función sexual. Los resultados reportan una prevalencia de dispareunia del 59,37\%, siendo más prevalente en mujeres sometidas a episiotomía (OR: 68,17; IC95 \%: 60,57-79,15). Respecto a la asociación de la episiotomía con la presencia de dispareunia se encontró asociación significativa con la episiotomía medio-lateral (OR: 8,17; IC95\%: 2,15-56,32), lesión del esfínter (OR: 7,32; IC95\%: 4,8612,75 ) y dehiscencia de la sutura (OR: 6,48; IC95\%: 4,92-14,28). Se observaron como factores de riesgo: antecedente de endometriosis (OR: 11,6; IC95 \%: 9,15-17,52), dolor pélvico crónico (OR: 3,56; IC95\%: 2,21-6,32) e ITS (OR: 2,67; IC95\%: 1,54-5,14), p=0,003). Se concluye que la prevalencia de dispareunia en mujeres con parto vaginal, atendidas en Armenia, varía en función de la realización de episiotomía, asociándose tanto a las complicaciones de esta como a otros antecedentes.

\section{ABSTRACT}

Keywords: Dyspareunia; Prevalence; Natural Childbirth; Women; Episiotomy.
A cross-sectional study was conducted to determine the prevalence of dyspareunia in women with vaginal delivery, to assess possible risk factors and the association with episiotomy. Women attended for vaginal delivery, between 2012 and 2017, were admitted in two high complexity clinics in Armenia (Colombia). The Female Sexual Function Index (IFFS-6) questionnaire was used to assess sexual function. The results report a prevalence of dyspareunia of $59.37 \%$, prevalent in women undergoing episiotomy (OR: 68,17; IC95\%: 60,57-79,15). Regarding the association of episiotomy with the presence of dyspareunia, a significant association was found with mid-lateral episiotomy (OR: 8,17; IC95\%: 2,1556,32), sphincter injury (OR: 7,32; IC95\%: 4,86-12,75), and suture dehiscence (OR: 6,48; IC95\%: 4,9214,28). The following were observed as risk factors: history of endometriosis (OR: 11,6 ; IC95\%: 9,1517,52), chronic pelvic pain (OR: 3,56; IC95\%: 2,21-6,32) and STIS (OR: 2,67; IC95\%: 1,54-5,14), p=0,003). It is concluded that the prevalence of dyspareunia in women with vaginal delivery, attended in Armenia, varies depending on the episiotomy performance. Both are associated with the complications of this and other backgrounds. 


\section{INTRODUCCIÓN}

La dispareunia en la mujer se define como el dolor recurrente o persistente asociado con la actividad coital, que causa una marcada angustia o un conflicto interpersonal ${ }^{1-3}$.

En la población general, la prevalencia de dispareunia oscila entre $9,3 \%$ y $70 \%$, mientras que en el postparto varía de $41 \%$ a $83 \%$ (a los 2-3 meses) y hasta $64 \%$ a los 6 meses; dependiendo, entre otras variables, de la edad de las mujeres, del país y de cómo se haya definido la dispareunia ${ }^{4-6}$.

La dispareunia posterior al parto es una condición usual6,7 que se asocia con dificultades obstétricas y ginecológicas, afectando de forma negativa la calidad de vida de las mujeres que la padecen ${ }^{7}$.

En Boston (Massachusetts), Signorello et $a l^{8}$ en un grupo de 921 mujeres primíparas reportaron que, después del parto vaginal, el $41 \%$ y el $22 \%$ de las mujeres, a los tres y seis meses, respectivamente, experimentaron dispareunia8. Esta se debe a estiramiento perineal, laceraciones obstétricas, parto instrumentado o uso de la episiotomía, ya que pueden resultar en cicatrización esclerótica6.

El parto instrumentado y la extensión de la laceración o el desgarro obstétrico son factores predictivos de dolor perineal en el postparto, constituyéndose en una causa de afectación de la vida sexual de la mujer ${ }^{6,8,9}$.

En el período postparto, especialmente en las mujeres lactantes, se presenta una disminución de los niveles de estrógenos circulantes, lo que conduce a sequedad vaginal y dispareunia ${ }^{10}$.

El uso rutinario y sistemático de la episiotomía no ha demostrado mayores ventajas en varios estudios, en comparación con la episiotomía restringida a la hora de prevenir el trauma perineal durante la atención del parto ${ }^{11,12}$, a fin de evitar la dispareunia postparto. Sin embargo, en contraposición, una creciente ola de investigadores ha informado la asociación existente entre la episiotomía y la dispareunia ${ }^{13-16}$.
La dispareunia ha sido evaluada en varios grupos de mujeres, principalmente en la población general; sin embargo, no se ha publicado en el Eje Cafetero nada relacionado con la dispareunia postparto. Su búsqueda e identificación precoz de los trastornos en la función sexual de la mujer en puerperio, al igual que los factores asociados, facilitan el ofrecimiento de la oportuna ayuda en la mujer con dispareunia, evitando la progresión de dicha dificultad sexual. Por eso, el objetivo de este estudio consistió en determinar la prevalencia de la dispareunia en mujeres con parto vaginal y evaluar los posibles factores de riesgo y su asociación con la episiotomía en una población de mujeres de Armenia (Quindío), Colombia.

\section{MATERIALES Y MÉTODOS}

\section{Tipo de investigación}

Se hizo un estudio observacional descriptivo de corte transversal en el cual se incluyeron mujeres con parto vaginal, con producto vivo, que fueron atendidas desde el 01 de abril 2012 al 31 de marzo del 2017 en dos clínicas de alta complejidad. Ambos centros son de referencia en el departamento del Quindío y norte del departamento del Valle del Cauca (Colombia), pues se allí atiende población perteneciente al régimen contributivo y subsidiado en el sistema de seguridad social vigente en el país. A dichas mujeres se les pidió diligenciar el cuestionario Índice de Función Sexual Femenina abreviado (IFFS-6), como instrumento de evaluación.

Se incluyeron a todas las mujeres mayores de 18 años en edad fértil que consultaron el servicio de ginecología y obstetricia, que cursaban el último trimestre del embarazo, en trabajo de parto o que habían tenido parto vaginal en los últimos dos meses a la consulta. Se excluyeron partos por cesárea, partos atendidos en casa y partos terminados en feto muerto; también las mujeres que se negaron a participar y las que no suministraron la información completa 0 se mudaron de ciudad. 


\section{Participantes}

El tamaño de la muestra no se calculó, sino que se hizo un muestreo no probabilístico por conveniencia a partir del universo de mujeres pertenecientes al programa de consulta prenatal y anticoncepción planificación familiar. El muestreo se hizo de forma consecutiva.

\section{Instrumentos}

El IFFS-617 es un cuestionario de seis preguntas que sirve para evaluar la función sexual en las últimas cuatro semanas, además de ser útil para identificar la presencia de trastornos sexuales. Se basa en la versión original de 19 preguntas del Female Sexual Function Index (FSFI)18. Considera seis dominios: deseo, excitación, lubricación, orgasmo, satisfacción y dolor. Cada pregunta ofrece cinco o seis opciones de respuesta que se califican de 0 a 5 . Los ítems de deseo y satisfacción se califican en una escala Likert de 5 puntos que va de 1 a 5 , y los otros ítems se califican en una escala Likert de 6 puntos que van de 0 a 5 . El puntaje total de la escala es la suma aritmética de los dominios. Los puntajes totales van de 2 a 30. Entre más alto es el puntaje, mejor es la sexualidad. Se tiene riesgo de disfunción sexual con un puntaje inferior a 19. Este instrumento ha demostrado ser válido y fiable $[\alpha$ de Cronbach $=$ $0,78]$, además de que cuenta con una sensibilidad y especificidad superior al $94 \%$ con valor de $\mathrm{p}<0,00117$.

\section{Procedimiento}

A las mujeres que consultaron a control prenatal del último trimestre, que ingresaban en trabajo de parto o habían tenido parto vaginal en los últimos dos meses, se les solicitó autorización para participar en la investigación mediante firma del consentimiento informado. Una vez autorizaron el ingreso al estudio se procedió a la revisión de la información de los datos sociodemográficos, antecedentes sexuales y demás. A los dos meses del parto se les suministró el cuestionario IFFS- 6 para la identificación del dolor coital. Todas las mujeres que cumplieron con los criterios de elegibilidad fueron incluidas.

Los desenlaces principales considerados fueron la dispareunia o dolor pélvico y perineal, así como la realización de episiotomía, técnica de sutura empleada y la puntuación del IFFS-6.

La recolección del reporte del cuestionario IFFS- 6 se acompañó de una entrevista de las pacientes por personal entrenado para ello, en la que se incluía otro cuestionario semiestructurado, diseñado exclusivamente para esta investigación, el cual había sido revisado previamente por expertos. Adicionalmente, se tomó información de la historia clínica de cada una de las instituciones.

Se establecieron dos grupos: parto vaginal asistido con episiotomía y parto vaginal sin episiotomía.

Variables medidas: se tuvieron en cuenta variables socio-demográficas (edad, raza, estado civil, ocupación, lugar de procedencia, escolaridad, religión), talla, peso, índice de masa corporal (IMC), hábitos (ingesta de alcohol, tabaquismo, consumo de sustancias psicoactivas y cafeína), actividad física; y variables de salud sexual y reproductiva: edad de la menarquia, edad de inicio de vida sexual y obstétrica, número de parejas sexuales, uso de preservativo, métodos anticonceptivos, masturbación, sexo oral, coito -vaginal o anal-, frecuencia promedio de relaciones sexuales semanales, antecedente de abuso sexual o violencia sexual, tiempo de convivencia en pareja. También se tuvieron en cuenta antecedentes personales patológicos (infecciones vaginales, urinarias durante el embarazo e ITS) y quirúrgicos.

\section{Análisis estadístico}

Los análisis fueron realizados usando el paquete estadístico STATA ${ }^{\circledR}$ versión 11.0. Se calculó la media y la desviación estándar (DS) o las medianas y el rango según el tipo de variable, y se calcularon proporciones para las variables categóricas. La comparación de las variables categóricas se realizó con la prueba de $\mathrm{X} 2$ o la prueba exacta de Fisher, y para las variables continuas se utilizó la prueba t de Student o Wilcoxon Mann Whitney, dependiendo de la normalidad de la muestra. Los Odds Ratio (OR) y su respectivo Intervalo de Confianza $95 \%$ (IC $95 \%$ ) fueron calculados. Valores de $p \leq 0,05$ fueron considerados estadísticamente significativos. 


\section{Declaración sobre aspectos éticos}

Este estudio adaptó las normas establecidas en la Declaración de Helsinki de 1975 y la Resolución 8430 de 1993 del Ministerio de Salud de Colombia para la investigación con seres humanos. Se presentó el consentimiento informado y se protegió el bienestar e integridad de los participantes a través de la confidencialidad y el derecho a la no participación, retiro y devolución de resultados. La investigación fue avalada por el comité de ética de la Universidad del Magdalena REC-029-14.

\section{RESULTADOS}

Se seleccionaron 6417 mujeres para un seguimiento de 60 meses. Se excluyeron 318 (4,95\%) por no cumplir los criterios de inclusión, 72 (1,12\%) se negaron a participar, $93(1,44 \%)$ no suministraron la información completa, $48(0,74 \%)$ se mudaron de ciudad. Al final, participaron en la investigación 5886 $(91,72 \%)$ mujeres.

La media de edad de la población total de las mujeres fue de 26,5 $\pm 3,16$ años, mediana de 21 años (rango entre 18 y 42); mientras que la edad media de las parejas fue de $29,7 \pm 4,29$ años, mediana de 24 años (rango entre 21 y 57). El tiempo de convivencia en pareja reportó una media de 3,78 $\pm 1,89$ años, mediana de 3 años (rango 0 y 9). En relación con las características basales, la edad, el peso, la talla, el IMC, la paridad, y las características sociodemográficas, en general, no se mostraron diferencias significativas (Tabla 1).

Tabla 1. Características sociodemográficas de mujeres con dispareunia postparto, Armenia, Colombia, 20122017.

\begin{tabular}{|c|c|c|c|}
\hline & $\begin{array}{l}\text { Con episiotomía } \\
\quad(\mathrm{n}=3657)\end{array}$ & $\begin{array}{l}\text { Sin episiotomía } \\
\quad(n=2229)\end{array}$ & $p$ \\
\hline Edad años & $27,3 \pm 2,4$ & $26,7 \pm 3,7$ & NS \\
\hline Peso $(\mathrm{Kg})$ & $63,1 \pm 9,6$ & $63,4 \pm 8,5$ & 0,807 \\
\hline Talla & $1,58 \pm 10,9$ & $1,57 \pm 11,6$ & 0,792 \\
\hline $\operatorname{IMC}\left(\mathrm{Kg} / \mathrm{m}^{2}\right)$ & $25,3 \pm 4,7$ & $25,6 \pm 3,9$ & 0,681 \\
\hline \multicolumn{4}{|l|}{ Paridad } \\
\hline Primíparas & $1206(32,97 \%)$ & $741(33,24 \%)$ & \multirow[t]{2}{*}{0,834} \\
\hline Multíparas & $2451(67,02 \%)$ & $1488(66,75 \%)$ & \\
\hline \multicolumn{4}{|l|}{ Estado civil } \\
\hline Casadas \% & $1467(40,11 \%)$ & $849(38.08 \%)$ & \multirow{3}{*}{0,819} \\
\hline Unión libre \% & $1731(47,33 \%)$ & $1092(48,99 \%)$ & \\
\hline Solteras \% & $459(12,55 \%)$ & $288(12,92 \%)$ & \\
\hline \multicolumn{4}{|l|}{ Procedencia } \\
\hline Rural \% & $1200(32,81 \%)$ & $579(25,97 \%)$ & \multirow[b]{2}{*}{0,723} \\
\hline Urbana \% & $2457(67,18 \%)$ & $1650(74,02 \%)$ & \\
\hline \multicolumn{4}{|l|}{ Ocupación } \\
\hline Ama de casa \% & $2064(56,43 \%)$ & $1257(56,39 \%)$ & \multirow[t]{2}{*}{0,804} \\
\hline Empleada & $1593(43,56 \%)$ & $972(43,60 \%)$ & \\
\hline \multicolumn{4}{|l|}{ Raza } \\
\hline $\begin{array}{l}\text { Afrocolombianas / } \\
\text { Indígenas }\end{array}$ & $1278(34,9 \%)$ & $717(32,16 \%)$ & \multirow[t]{2}{*}{0,741} \\
\hline Hispánicas / Mestizas & $2379(65,05 \%)$ & $1512(67,83 \%)$ & \\
\hline \multicolumn{4}{|l|}{ Estrato socioeconómico } \\
\hline Bajo & $1812(49,54 \%)$ & $1140(51,14 \%)$ & \multirow{3}{*}{0,957} \\
\hline Medio & $1143(31,25 \%)$ & $627(28,12 \%)$ & \\
\hline Alto & $702(19,19 \%)$ & $462(20,72 \%)$ & \\
\hline
\end{tabular}


Respecto a los antecedentes quirúrgicos, se destacan apendicectomía (15,13\%), colecistectomía (11,92\%), amigdalectomía (8,97\%), salpingectomía unilateral $(5,96 \%)$ y ooforectomía unilateral $(2,95 \%)$ en el total de la población.

En cuanto a los antecedentes patológicos se destacan HTA $(12,94 \%)$, diabetes $(10,24 \%)$, hipotiroidismo (8,91\%), endometriosis $(13,35 \%)$, dolor pélvico crónico $(15,54 \%)$, colitis ulcerativa y otros trastornos intestinales (14,98\%). El 38,99\% relataron haber padecido infecciones urinarias durante el embarazo.

Respecto al antecedente de ITS, el 20,99\% del total de las participantes afirmó haber recibido tratamiento por esta causa. Las infecciones vaginales se hicieron presentes en el $82,31 \%$ $(n=4845 / 5886),[31,95 \% \quad(n=1548 / 4845)$ vaginosis bacteriana y $68,04 \%$ ( $n=3297 / 4845)$ vaginitis por cándida spp]. La infección por herpes genital representó el 45,14\%, seguido por VPH 35,43\%, sífilis $11,89 \%$, VIH 3,88\% e infección gonocócica $2,91 \%$. El $32,31 \%$ refirió haber recibido antibioticoterapia por al menos un episodio de Enfermedad Pélvica Inflamatoria. El 27,11\% afirmó que nunca había usado preservativo.

Alrededor de 1 de cada 3 mujeres tenía entre 18 y 24 años, 21,04\% tenían educación primaria o menos y $35,98 \%$ había terminado o cursado por lo menos un año de educación secundaria, mientras que solo el $18,55 \%$ eran profesionales, aunque $41,43 \%$ reportaron estar empleadas. 4 de cada 6 mujeres declaró como actividad principal estar dedicada al hogar. El 76,29\% profesaban la religión católica, seguida de los testigos de Jehová $(12,18 \%)$.

El tabaquismo estuvo presente en el $25,63 \%$, mientras el consumo de alcohol reportó el $86,69 \%$. El consumo de sustancias psicoactivas se detectó en el $5,14 \%$ y el de café en el $42,25 \%$. El sedentarismo se encontró en el $48,57 \%$. La actividad física regular ( $\geq 4$ veces a la semana) se observó en el $26,96 \%$.

La edad media de la menarquia fue a los $13,24 \pm 1,58$ años, mediana de 11 años (rango entre 10 y 17). La edad de inicio de las relaciones sexuales en la población global fue de $16,47 \pm 1,53$ años, mediana de 16 años (rango entre 12 y 21 años). El 52,95\% de las mujeres iniciaron sus relaciones sexuales antes de los 15 años. Respecto al número de parejas sexuales se obtuvo una mediana de 9 (rango entre 3 $y \geq 27$ ). El $87,05 \%$ de las mujeres refirieron una única pareja sexual en los últimos 12 meses.

La edad del primer parto arrojó una media de $17,49 \pm 1,38$ años, mediana de 15 años (rango entre 14 y 27 años). La paridad arrojó una mediana de 3 hijos ( 2 vía vaginal y 1 vía cesárea por mujer), y un rango entre 2 y 9 hijos. El 17,94\% de ellas tenía antecedente de aborto, de las cuales el 9,37\% manifestó una interrupción voluntaria del embarazo por diferentes causas, mientras el 6,42\% reconoció haberse realizado un aborto en condiciones clandestinas. El embarazo ectópico se encontró en el $6,37 \%$ del total de las participantes. El 88,63 \% de los embarazos fueron no planeados.

El 17,99\% de la población total de las mujeres eran primíparas. El 29,96\% fueron madres adolescentes, de las cuales el $57,31 \%$ tuvo su primer parto por cesárea. El 1,98\% tenían antecedente de parto instrumentado con fórceps y $11,92 \%$ con espátulas. Se observó una prevalencia de desgarros de tercer y cuarto grado del 2,95\%.

Las relaciones sexuales vaginales son la práctica más frecuente $(100 \%)$, seguido por las relaciones sexuales orales $(89,95 \%)$ y anales $(11,31 \%)$. Luego del parto, el 48,52\% prefería la masturbación sobre la penetración. El 83,79\% afirmó disfrutar más que le practicaran sexo oral.

La media de tiempo para que las mujeres reanudaran sus relaciones sexuales coitales después del parto osciló entre 6 y 9 semanas con una mediana de 7 semanas.

Se encontró que solo el $21,28 \%$ de las mujeres solteras usaron condón en todas las relaciones sexuales después del puerperio. La anticoncepción se encontró en el $89,95 \%$, siendo los métodos hormonales los preferidos $(76,26 \%)$, destacándose la píldora con el $68,94 \%$, seguida del implante $(20,28 \%)$, inyección $(8,39 \%)$ y por último el parche (2,37\%). Entre los métodos no hormonales, el DIU de $\mathrm{Cu}$ era usado por el $5,77 \%$ de las mujeres, el 
$16,94 \%$ se había practicado un método definitivo y solo el 1,01\% utilizaba métodos naturales.

A la pregunta ¿cuántas veces tuvo relaciones sexuales la semana pasada? (periodo definido como el lapso de los siete días anteriores), el 88,98\% contestó 3 veces, arrojando una mediana de 2 relaciones sexuales por semana (rango entre 0 y 5 ).

A un total de 3657 de ellas $(62,13 \%)$ se les practicó episiotomía. Del total de mujeres participantes del estudio se les diagnosticó dispareunia a 3495 (59,37\%); siendo mayor el número cuando el parto fue asistido con episiotomía, lo que implica una prevalencia de 68,17\% (IC95\%: 60,57-79,15), mientras que en las mujeres cuyo parto fue atendido sin la asistencia de la episiotomía la prevalencia fue del 15,34 \% (IC95\%: 10,38-27,26).
Al evaluar la puntuación del IFFS-6, el dominio más alterado fue el dolor, observándose que el 59,37\% $(n=3495)$ de las mujeres presentaban dispareunia en el postparto. La lubricación vaginal durante el coito se afectó en el 33,13\% (Figura 1).

Del total de mujeres a las que se les realizó episiotomía, al $20,18 \%$ se les practicó mediana y al $79,81 \%$ medio-lateral. Se observó menor dolor en las mujeres suturadas con técnica continua en relación con la técnica discontinua (OR: 0,47; IC95\%: $0,26-0,83)$. Adicionalmente, el $8,97 \%$ de las pacientes evaluadas reportaron dispareunia en algún momento del embarazo. No se presentaron casos de fístulas.

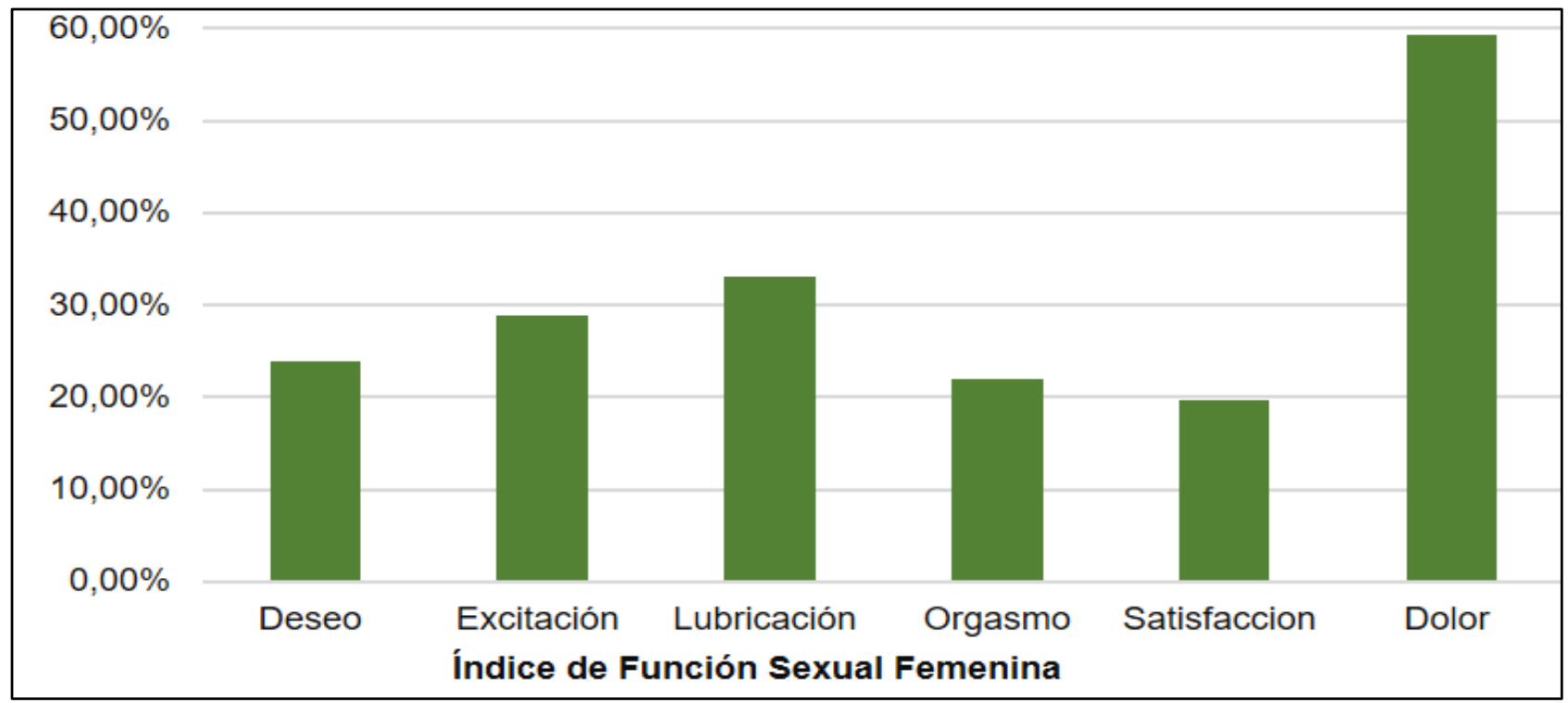

Figura 1. Prevalencia de disfunciones sexuales en mujeres con parto vaginal, Armenia, Colombia, 2012-2017.

La dispareunia fue más prevalente en el grupo de parto asistido por episiotomía (OR: 2,01; IC95\%: $1,47-2,85$ ) y parto instrumentado (OR: 1,62 ; IC95\%: 1,26-2,82); por otro lado, las mujeres con antecedente de dolor pélvico crónico y las mujeres con antecedente de ITS mostraron un mayor riesgo de dispareunia en comparación con las mujeres sin estos antecedentes (OR: 3,56; IC95\%: 2,21-6,32 y OR:2,67; IC95\%: 1,54-5,14, $\quad \mathrm{p}=0,003$, respectivamente). Por otra parte, la dispareunia se encontró 11,6 veces más en mujeres con endometriosis (IC 95\%: 9,15-17,52) al compararlas con las mujeres que no reportaron tener dicha condición. A la vez, tener un antecedente de infección urinaria recurrente y fumar incrementó la probabilidad de presentar dispareunia (Tabla 2). 
Tabla 2. Factores de riesgo para dispareunia postparto, en 5886 mujeres de Armenia, Colombia, 2012-2017.

\begin{tabular}{|l|c|c|c|}
\hline Variables asociadas & $\begin{array}{c}\text { Con episiotomía } \\
(\mathrm{n}=3657)\end{array}$ & $\begin{array}{c}\text { Sin episiotomía } \\
(\mathrm{n}=2229)\end{array}$ & $\begin{array}{c}\text { Razón de } \\
\text { Prevalencia (IC 95\%) }\end{array}$ \\
\hline $\begin{array}{l}\text { Uso de un dispositivo } \\
\text { intrauterino }\end{array}$ & $22 \%$ & $17 \%$ & $1,17(0,87-1,74)$ \\
\hline $\begin{array}{l}\text { Antecedentes de } \\
\text { infecciones de transmisión } \\
\text { sexual }\end{array}$ & $45 \%$ & $14 \%$ & $2,67(1,54-5,14)$ \\
\hline $\begin{array}{l}\text { Antecedentes de } \\
\text { enfermedad inflamatoria } \\
\text { pélvica }\end{array}$ & $20 \%$ & $32 \%$ & $1,14(0,81-1,65)$ \\
\hline $\begin{array}{l}\text { Antecedentes de colitis } \\
\text { ulcerativa y otros } \\
\text { trastornos intestinales }\end{array}$ & $3 \%$ & $2,7 \%$ & $1,89(0,84-1,53)$ \\
\hline $\begin{array}{l}\text { Antecedentes de } \\
\text { endometriosis dolor }\end{array}$ & $12,87 \%$ & $6,99 \%$ & $11,6(9,15-17,52)$ \\
\hline $\begin{array}{l}\text { Antecedente de délvico crónico } \\
\text { périnaria }\end{array}$ & $20,83 \%$ & $15,16 \%$ & $3,56(2,21-6,32)$ \\
\hline $\begin{array}{l}\text { Infección } \\
\text { recurrente }\end{array}$ & $33 \%$ & $17 \%$ & $2,04(1,442,91)$ \\
\hline Tabaquismo & $39 \%$ & $18 \%$ & $2,16(1,65-3,48)$ \\
\hline Episiotomía & $39 \%$ & $15 \%$ & $2,01(1,472,85)$ \\
\hline Parto instrumentado & $36 \%$ & $12 \%$ & $1,62(1,26-2,82)$ \\
\hline
\end{tabular}

Se encontró un aumento de dispareunia en las mujeres con antecedente de violencia sexual de niñas, ya que, al compararlas con las mujeres que no informaron o negaron violencia sexual, las que reportaron violencia antes de los 12 años estuvieron cuatro veces más predispuestas a expresar dispareunia (OR: 4,23; IC95\%:1,74-3,98; $p=0,001$ ). A su vez, las mujeres abusadas por sus propias parejas (6,37 \%) fueron susceptibles de dispareunia casi dos veces más (OR: 1,87; IC95\%: 1,05-5,13).
Respecto a la asociación de la episiotomía y la dispareunia, se encontró asociación significativa con la episiotomía medio-lateral (OR: 8,17; IC95\%: 2,1556,32), lesión del esfínter (OR: 7,32; IC95\%: 4,86$12,75)$ y dehiscencia de la sutura (OR: 6,48; IC95\%: 4,92-14,28) (Tabla 3).

Tabla 3. Criterios de asociación de la dispareunia postparto con la episiotomía, en 5886 mujeres de Armenia, Colombia, 2012-2017.

\begin{tabular}{|l|c|c|}
\hline \multicolumn{1}{|c|}{ Factores asociados } & OR & IC 95\% \\
\hline Episiotomía medio-lateral & 8,17 & $2,15-56,32$ \\
\hline Lesión del esfínter & 7,32 & $4,86-12,75$ \\
\hline Dehiscencia de la sutura & 6,48 & $4,92-14,28$ \\
\hline Formación de granulomas & 4,89 & $3,54-12,42$ \\
\hline Dos o más episiotomías & 4,05 & $3,48-7,59$ \\
\hline Estrechamiento del introito vaginal & 4,77 & $3,72-6,54$ \\
\hline Infección de la episiorrafia & 4,62 & $3,17-8,25$ \\
\hline Episiotomía mediana & 3,77 & $1,68-7,14$ \\
\hline
\end{tabular}


Las mujeres reportaron la dispareunia como una condición dolorosa de intensidad leve en el 17,97\%, leve a moderada en el $27,26 \%$, moderada en el $38,72 \%$ y severa en el $16,04 \%$, situación que alteró su ciclo de relación sexual con significativos cambios en la frecuencia coital. El $64,17 \%$ reportó haber disminuido el número de relaciones sexuales, lo cual afectaba su relación de pareja en un $78,52 \%$. Sin embargo, únicamente el $20,98 \%$ comentó haberlo discutido con su médico.

\section{DISCUSIÓN}

A pesar del significativo impacto de la función sexual en la vida de las personas, los profesionales de la salud a menudo descuidan su relevancia en la mujer después del parto. Se ha descrito que, en el postparto, hay afectación en todos los dominios del ciclo de la respuesta sexual, entre ellos la dispareunia, la disminución de la lubricación, la dificultad para alcanzar el orgasmo, la pérdida del deseo sexual, el sangrado o la irritación vaginal ${ }^{7}$. En el presente estudio se encontró una prevalencia de dispareunia en mujeres con parto vaginal del $59,37 \%$, y varió en función de la realización de episiotomía $(68,17 \%$ vs. $15,34 \%)$ y de las complicaciones asociadas a la misma. Se observó asociación de la dispareunia con la episiotomía medio-lateral (OR: 8,17; IC95\%: 2,15-56,32), la lesión del esfínter (OR: 7,32; IC95\%: 4,86-12,75) y la dehiscencia de la sutura (OR: 6,48; IC95\%: 4,9214,28) (Tabla 3).

En este estudio se encontró que la media de tiempo para que las mujeres reanudaran sus relaciones sexuales coitales osciló entre 6 y 9 semanas después del parto, mostrando concordancia con los resultados de Grudzinskas ${ }^{19}$ en una población de Inglaterra, los cuales reportaron un promedio de 5 semanas después del parto. Por su parte, Connolly et al20, en Carolina del Norte, describieron que las mujeres reanudaron las relaciones sexuales a las 6 semanas.

Los resultados del estudio reportan una prevalencia de dispareunia del 59,3\%, superior al 37,5\% descrito por Alkareem et $a{ }^{21}$ en Sudán. También son superiores al $40,7 \%$ informado por Lagaer et $a l^{22}$ en Bélgica y al $43,4 \%$ en la cohorte prospectiva multicéntrica de McDonald et $a l^{23}$, en Australia. No se pudo identificar la causa por la cual los hallazgos resultaron inferiores a los reportados en el presente estudio.

La dispareunia en la población de mujeres sometidas a episiotomía fue alta (OR: 68,17; IC95\%: 60,57-79,15); de tal manera que 6 de cada 10 mujeres a las cuales se les realizó episiotomía manifestó dolor postparto durante el coito vaginal. Estos hallazgos son superiores a los reportados por Fahmy et al ${ }^{24}$ en Egipto, los cuales describen que el $49 \%$ de las pacientes presentaron dolor significativo después del parto, y al 21,2\% descrito por AlligoodPercoco et $a l^{25}$ en Hershey (USA). Sin embargo, es inferior a los resultados de Andrews et $a l^{26}$, los que reportaron en un grupo de 254 mujeres del Reino Unido un $92 \%$ de dolor luego del primer parto vaginal, lo cual estuvo asociado a traumatismos perineales, lo que se alinea con los hallazgos de la presente investigación.

De acuerdo con los resultados de este estudio, los factores de riesgo asociados con la dispareunia son concordantes con los reportados por Abd. Alkareem et $a l^{21} \mathrm{y}$ con los informados por Gutzeit et $a l^{6}$, cuyos resultados estuvieron relacionados tanto con las complicaciones de la episiotomía, como de sus posibles secuelas. En la presente investigación, la episiotomía y las complicaciones asociadas a ella, junto con la violencia sexual y a otros antecedentes (Tabla 2), explican los altos porcentajes de dispareunia reportados.

La violencia sexual hacia la mujer, infligida por la pareja, reporta cifras alarmantes en esta población $(6,37 \%)$, prevalencia similar a la reportada por Espitia $^{27}$ en un estudio realizado en 12 ciudades de Colombia, pero inferior al $19,35 \%$ publicado en un grupo de mujeres del Quindío ${ }^{28}$. Desafortunadamente, a esta situación se le da una percepción de fenómeno común (mujeres forzadas a tener relaciones sexuales con su pareja cuando ellas no lo deseaban), destacándolo como un factor de riesgo para dispareunia. Esto lo señala Golding et $\mathrm{al}^{29}$ en California, al afirmar que las mujeres con afecciones ginecológicas comunes tienen un riesgo sustancialmente mayor de tener un historial de agresión sexual; similar a lo reportado por 
Chapman ${ }^{30}$ en EE. UU., quien reveló que el $61 \%$ de las mujeres víctimas de abuso sexual tenían disfunciones sexuales y problemas ginecológicos.

En cuanto a los criterios de asociación de la dispareunia con la episiotomía, Solana-Arellano et $\mathrm{al}^{31}$, en México, documentaron dicha asociación con las complicaciones de la episiotomía. Por su parte, Chayachinda et $a l^{32}$ aseguran que la dispareunia es común en mujeres primíparas tailandesas con antecedente de episiotomía, las que tienen una reanudación más lenta de la función sexual normal. Por otra parte, la dehiscencia y el estrechamiento del introito vaginal, posterior a la episiorrafia, provocan mayor riesgo de dispareunia, según lo reportan Hartmann et $a l^{33}$, en Carolina del Norte, siendo concordante con lo que se describe en este estudio.

A pesar de las recomendaciones de no justificar la realización sistemática de la episiotomía ${ }^{34-36}$, en la práctica clínica las cosas son diferentes, dado que se hace rutinariamente. La investigación arrojó una tasa de episiotomía de $62,13 \%$, porcentaje superior al $41,5 \%$ presentado por Rasouli et $\left.a\right|^{37}$, pero similar al $65,4 \%$ publicado por Tefera et $\left.a\right|^{38}$, aunque inferior al 93,3\% descrito por Kartal et $\left.a\right|^{39}$.

Se observó menor dolor en las mujeres suturadas con técnica continua en relación con la técnica discontinua (OR: 0,47; IC95\%: 0,26-0,83), coincidiendo con otros reportes ${ }^{40,41}$; mientras que el uso de las espátulas o fórceps se asoció con un mayor grado de desgarro y con mayor presencia y severidad de la dispareunia tal como lo reporta Signorello et $a l^{8}$ en Boston. Estos últimos concluyen que las mujeres cuyos hijos nacieron asistidos por la episiotomía o instrumentos obstétricos estuvieron relacionados con la frecuencia o la gravedad de la dispareunia posparto.

Es incuestionable que en los partos cuyo periné permanece integro las mujeres presentan menor presencia de dispareunia ${ }^{8,35,38}$, por lo tanto, es imperioso minimizar el daño perineal en el cual incurre la mujer durante la atención del parto.

Los resultados de esta investigación muestran que la episiotomía puede causar dispareunia, condición que suele afectar negativamente la vida sexual de las mujeres: datos consistentes con las publicaciones de otros autores ${ }^{9,13,15,21}$. Por lo tanto, no se recomienda la episiotomía de rutina. A aquellas mujeres sometidas a episiotomía se les debe realizar un adecuado seguimiento y vigilancia de la episiorrafia en el puerperio, a fin de detectar o minimizar sus eventuales complicaciones.

Es preocupante la preferencia de los profesionales de la salud por la episiotomía medio-lateral, cuando es clara su asociación con la dispareunia (OR: 8,17; IC95\%: 2,15-56,32), lo cual la convierte en un indiscutible factor de riesgo. Sería conveniente analizar tanto el tipo de episiotomía a realizar, así como el tipo de sutura a emplear, puesto que algunos estudios señalan que la dispareunia es más frecuente con la episiotomía medio-lateral, en comparación con la episiotomía mediana (relación 2:1); además de que la técnica de sutura continua se asocia con menos dolor $(34,2 \%)$ frente a la sutura interrumpida $(65,8 \%)^{42,43}$.

De acuerdo con los resultados de este estudio, aún existe una gran necesidad de educación continua a la hora de atender el parto vaginal, de acuerdo con las pautas aceptadas basadas en la evidencia, a fin de lograr la mejor atención y beneficios para las pacientes.

La decisión de realizar o evitar la episiotomía está relacionada con la situación clínica real de cada mujer. Su adecuado enfoque permite tener menos complicaciones relacionadas con el parto y así lograr una buena calidad de vida y función sexual satisfactoria después del parto. Las indicaciones estarían reservadas y determinadas, caso por caso, por el equipo sanitario.

Las situaciones en las que se puede considerar una episiotomía incluyen indicaciones maternas o fetales: asistencia de un parto vaginal instrumentado, distocia de hombros o pérdida del estado del bienestar fetal (sufrimiento fetal) ${ }^{44,45}$, a fin de facilitar o agilizar partos difíciles o evitar laceraciones maternas graves ${ }^{46}$. La técnica continua, utilizando suturas absorbibles durante la reparación de la episiorrafia, es el enfoque recomendado, ya que reduce la necesidad de una extracción adicional en una fecha posterior, si fuera necesario ${ }^{45,47}$. 
Debido a los resultados y las publicaciones revisadas, es conveniente alinearse con la posición de Sultan et $a l^{44}$, quienes afirman que la Organización Mundial de la Salud (OMS) tiene que establecer el papel de la episiotomía a la hora de la atención del parto vaginal.

Se finaliza reconociendo que todas las mujeres experimentan dispareunia ocasional en algún momento de su vida, sin embargo, lo indiscutible es que después del parto la prevalencia es significativamente mayor, tal como se ha demostrado en esta investigación. El infortunio de la dispareunia resulta en ser poco informada por las mujeres y no investigada por los médicos.

Entre las debilidades del estudio se destaca que no se contó con evaluaciones clínicas previas de la dispareunia en las mujeres estudiadas; otra es que el examen perineal no se pudo realizar a todas las participantes antes del estudio. Por otro lado, algunas de las variables incluidas pueden desencadenar dispareunia por sí solas. Como fortalezas se señala que la investigación fue realizada con una selección significativa de participantes y que se siguió de la misma forma en que se podría hacer en cualquier lugar del país o el mundo, lo que garantiza la uniformidad al momento de realizar la evaluación, así como la replicación y la aplicación del estudio en otras instituciones.

Se puede concluir que, en Armenia, la prevalencia de dispareunia es alta $(68,17 \%)$ en mujeres sometidas a episiotomía durante el parto vaginal. Esta está asociada tanto a complicaciones de la episiotomía como a otros antecedentes. Se sugieren investigaciones en dicha línea de asociación y su relación con otros factores de riesgo, en especial lo relacionado con el aumento de la dispareunia y el dolor perineal postparto, frente al nexo con el tipo de episiotomía y técnica de sutura empleada.

\section{AGRADECIMIENTOS}

A los maestros de la sexología latinoamericana, los doctores Fernando José Bianco Colmenares, Adrián Sapetti y Rubén Hernández Serrano, brillantes psiquiatras sexólogos, por abrirnos paso en la investigación, en especial para el seguimiento y estudio del dolor presente durante el coito; así como al doctor Carlos Arturo Díaz Támara, por permitirme conocer el universo envuelto en el piso pélvico y por facilitarme presentar este modesto trabajo en el 5o Congreso Internacional de la Asociación Latinoamericana de Piso Pélvico (ALAPP - 2020). Gracias, maestros.

\section{DECLARACIÓN SOBRE CONFLICTO DE INTERESES}

Los autores declaran que no existe conflicto de intereses.

\section{REFERENCIAS BIBLIOGRÁFICAS}

1. Rogers RG, Pauls RN, Thakar R, Morin M, Kuhn A, Petri E, Fatton B, Whitmore K, Kingsberg SA, Lee J. An international Urogynecological association (IUGA)/international continence society (ICS) joint report on the terminology for the assessment of sexual health of women with pelvic floor dysfunction. Int Urogynecol J. 2018; 29(5):647-66. Doi: https://doi.org/10.1007/s00192-018-3603-9

2. Orr N, Wahl K, Joannou A, Hartmann D, Valle L, Yong $P$, et al. Deep Dyspareunia: Review of Pathophysiology and Proposed Future Research Priorities. Sex Med Rev. 2018; 8(1):3-17. Doi: https://doi.org/10.1016/j.sxmr

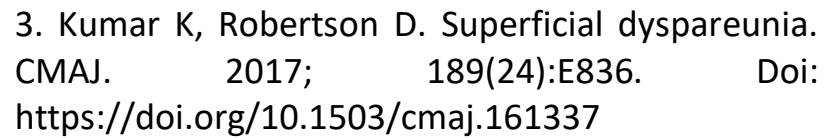

4. Polland AR, Davis M, Zeymo A, Iglesia CB. Association between comorbidities and female sexual dysfunction: findings from the third National Survey of Sexual Attitudes and Lifestyles (Natsal-3). Int Urogynecol J. 2019; 30(3):377-83. Doi: https://doi.org/10.1007/s00192-018-3739-7

5. Wolpe RE, Zomkowski K, Silva FP, Queiroz APA, Sperandio FF. Prevalence of female sexual dysfunction in Brazil: A systematic review. Eur J Obstet Gynecol Reprod Biol. 2017; 211:26-32. Doi: https://doi.org/10.1016/j.ejogrb.2017. 01.018 
6. Gutzeit O, Levy G, Lowenstein L. Postpartum Female Sexual Function: Risk Factors for Postpartum Sexual Dysfunction. Sex Med. 2020; 8(1):8-13. Doi: https://doi.org/10.1016/j.esxm.2019.10.005.

7. Serrano Drozdowskyj E, Gimeno Castro E, Trigo López E, Bárcenas Taland I, Chiclana Actis C. Factors Influencing Couples' Sexuality in the Puerperium: A Systematic Review. Sex Med Rev. 2020; 8(1):38-47. Doi: https://doi.org/10.1016/j.sxmr.2019.07.002

8. Signorello LB, Harlow BL, Chekos AK, Repke JT. Postpartum sexual functioning and its relationship to perineal trauma: a retrospective cohort study of primiparous women. Am J Obstet Gynecol. 2001; 184(5):881-8; discussion 888-90. Doi: http://dx.doi.org/10.1067/mob.2001.113855

9. McDonald EA, Gartland D, Small R, Brown SJ. Dyspareunia and childbirth: a prospective cohort study. BJOG. 2015; 122(5):672-9. Doi: http://dx.doi.org/10.1111/1471-0528.13263

10. Leeman LM, Rogers RG. Sex after childbirth: postpartum sexual function. Obstet Gynecol. 2012; 119(3):647-55.

Doi: http://dx.doi.org/10.1097/AOG.0b013e318247961 1

11. Jiang H, Qian X, Carroli G, Garner P. Selective versus routine use of episiotomy for vaginal birth. Cochrane Database of Systematic Reviews 2017, Issue 2. Art. No.: CD000081. Doi: http://dx.doi.org/10.1002/14651858.CD000081.pu b3

12. Carroli G, Mignini L. Episiotomy for vaginal birth. Cochrane Database Syst Rev. 2009 Jan 21;(1):CD000081. Doi: http://dx.doi.org/10.1002/14651858.CD000081.pu b2

13. Committee on Practice Bulletins-Obstetrics. ACOG Practice Bulletin No. 198: Prevention and Management of Obstetric Lacerations at Vaginal Delivery. Obstet Gynecol. 2018; 132(3):e87-e102. Doi: http://dx.doi.org/10.1097/AOG.000000000000284 1
14. Muraca GM, Liu S, Sabr Y, Lisonkova S, Skoll A, Brant $R$, et al. Episiotomy use among vaginal deliveries and the association with anal sphincter injury: a population-based retrospective cohort study. CMAJ. 2019; 191(42):E1149-E1158. Doi: https://doi.org/10.1503/cmaj.190366

15. Manresa M, Pereda A, Goberna-Tricas J, Webb SS, Terre-Rull C, Bataller E. Postpartum perineal pain and dyspareunia related to each superficial perineal muscle injury: a cohort study. Int Urogynecol J. 2020. Doi: http://dx.doi.org/10.1007/s00192-02004317-1

16. Marques Nascimento Silva A, Marques dos Santos L, Anny Costa Cerqueira E, Souza de Santana E, Carvalho, Silva Gomes Xavier A. Characterization of pain resulting from perineal trauma in women with vaginal delivery. Br J Pain. São Paulo. 2018; 1(2):158-62. Doi: https://doi.org/10.5935/25950118.20180030

17. Isidori AM, Pozza C, Esposito K, Giugliano D, Morano S, Vignozzi L, et al. Development and validation of a 6 -item version of the female sexual function index (FSFI) as a diagnostic tool for female sexual dysfunction. J Sex Med. 2010; 7(3):1139-46. Doi: $\quad$ http://dx.doi.org/10.1111/j.17436109.2009.01635.x

18. Rosen R, Brown C, Heiman J, Leiblum S, Meston $C$, Shabsigh $R$, et al. The Female Sexual Function Index (FSFI): a multidimensional self-report instrument for the assessment of female sexual function. J Sex Marital Ther. 2000; 26(2):191-208. Doi: http://dx.doi.org/10.1080/009262300278597

19. Grudzinskas JG, Atkinson L. Sexual function during the puerperium. Arch Sex Behav. 1984; 13(1):85-91.

Doi: http://dx.doi.org/10.1007/BF01542980.

20. Connolly A, Thorp J, Pahel L. Effects of pregnancy and childbirth on postpartum sexual function: a longitudinal prospective study. Int Urogynecol J Pelvic Floor Dysfunct. 2005; 16(4):263-7. Doi: http://dx.doi.org/10.1007/s00192-005-1293-6

21. Abd. Alkareem I, Ounsa M, Mohamed E, Mohamed E, Abdalla S. Prevalence and risk factors 
of postpartum dyspareunia at three months postdelivery in Sudanese women. Int J Reprod Contracept Obstet Gynecol. 2017; 6(7):2724-8. Doi: http://dx.doi.org/10.18203/2320-

1770.ijrcog20172901

22. Lagaert L, Weyers S, Van Kerrebroeck H, Elaut E. Postpartum dyspareunia and sexual functioning: a prospective cohort study. Eur J Contracept Reprod Health Care. 2017; 22(3):200-6. Doi: http://dx.doi.org/10.1080/13625187.2017.1315938

23. McDonald EA, Gartland D, Small R, Brown SJ. Dyspareunia and childbirth: a prospective cohort study. BJOG. 2015; 122(5):672-9. Doi: http://dx.doi.org/10.1111/1471-0528.13263

24. Fahmy MM, Al-Lakwa HE, Al-Halaby AE, AlSharkawy EA. Effect of different episiotomy techniques on perineal pain and sexual activity 3 months after delivery. Menoufia Med J. 2018; 31(4):1253-7.

Doi: http://www.mmj.eg.net/text.asp?2018/31/4/1253/ 252047

25. Alligood-Percoco NR, Kjerulff KH, Repke JT. Risk Factors for Dyspareunia After First Childbirth. Obstet Gynecol. 2016; 128(3):512-8. Doi: http://dx.doi.org/10.1097/AOG.000000000000159 0

26. Andrews V, Thakar R, Sultan AH, Jones PW. Evaluation of postpartum perineal pain and dyspareunia--a prospective study. Eur J Obstet Gynecol Reprod Biol. 2008; 137(2):152-6. Doi: http://dx.doi.org/10.1016/j.ejogrb.2007.06.005

27. Espitia-De La Hoz FJ. Prevalence and characterisation of sexual dysfunctions in women, in 12 Colombian cities, 2009-2016. Rev Colomb Obstet Ginecol 2018; 69(1):9-21. Doi: http://dx.doi.org/10.18597/rcog.3035

28. Espitia-De La Hoz FJ. Prevalence of genitourinary syndrome of menopause and impact on sexuality of women in Quindío (Colombia), 2013-2016. Rev Colomb Obstet Ginecol. 2018; 69(4):249-59. Doi: http://dx.doi.org/10.18597/rcog.3111
29. Golding JM, Wilsnack SC, Learman LA. Prevalence of sexual assault history among women with common gynecologic symptoms. Am J Obstet Gynecol. 1998; 179(4):1013-9. Doi: http://dx.doi.org/10.1016/S0002-9378(98)70208-X

30. Chapman JD. A longitudinal study of sexuality and gynecologic health in abused women. J Am Osteopath Assoc 1989; 89(5):619-24. Disponible en: https://pubmed.ncbi.nlm.nih.gov/2745177/

31. Solana-Arellano E, Villegas-Arrizón A, LegorretaSoberanis J, Cárdenas-Turanzas M, Enzaldo de la Cruz J, Andersson N. Dispareunia en mujeres después del parto: estudio de casos y controles en un hospital de Acapulco, México. Rev Panam Salud Publica. 2008; 23(1):44-51. Disponible en: https://www.scielosp.org/pdf/rpsp/v23n1/a06v23n 1.pdf

32. Chayachinda C, Titapant V, Ungkanungdecha A. Dyspareunia and sexual dysfunction after vaginal delivery in Thai primiparous women with episiotomy. J Sex Med 2015; 12:1275-82. Doi: http://dx.doi.org/10.1111/jsm.12860

33. Hartmann $K$, Viswanathan $M$, Palmieri $R$, Gartlehner G, Thorp J Jr, Lohr KN. Outcomes of routine episiotomy: a systematic review. JAMA. 2005; 293(17):2141-8. Doi: http://dx.doi.org/10.1001/jama.293.17.2141

34. Barua H, Alamgir S, Dewan G. Routine Episiotomy Practice: A Study from Bangladesh. Journal of Rangamati Medical College. 2018; 1(2):3$4 . \quad$ Disponible en: https://www.researchgate.net/publication/332948 285_Routine_Episiotomy_Practice_A_Study_from_ Bangladesh

35. Oraif A. Routine Episiotomy Practice at a Tertiary Care Center in Saudi Arabia. Open Journa of Obstetrics and Gynecology. 2016; 6:794-7. Doi: http://dx.doi.org/10.4236/ojog.2016.613097

36. Wu LC, Lie D, Malhotra R, Allen JC Jr, Tay JS, Tan TC, Ostbye T. What factors influence midwives' decision to perform or avoid episiotomies? A focus group study. Midwifery. 2013; 29(8):943-9. Doi: http://dx.doi.org/10.1016/j.midw.2012.11.017 
37. Rasouli M, Keramat A, Khosravi A, Mohabatpour Z. Prevalence and Factors Associated With Episiotomy in Shahroud City, Northeast of Iran. International Journal of Women's Health and Reproduction Sciences. 2016; 4(3):125-9. Doi: http://dx.doi.org/10.15296/ijwhr.2016.29

38. Tefera T, Kebede B, Mekonen T. Prevalence of episiotomy and factors associated with Practice of episiotomy at Saint Paul's Hospital Millennium Medical College: a cross sectional study. Ethiopian Journal of Reproductive Health. 2019; 11 (3):33-40. Disponible en:

http://ejrh.org/index.php/ejrh/article/view/306

39. Kartal B, Kızılırmak A, Calpbinici P, Demir G. Retrospective analysis of episiotomy prevalence. J Turk Ger Gynecol Assoc. 2017; 18(4):190-4. Doi: http://dx.doi.org/10.4274/jtgga.2016.0238

40. Kettle C, Hills RK, Jones P, Darby L, Gray R, Johanson $R$. Continuous versus interrupted perineal repair with standard or rapidly absorbed sutures after spontaneous vaginal birth: a randomised controlled trial. Lancet. 2002; 359(9325):2217-23. Doi: http://dx.doi.org/10.1016/S01406736(02)09312-1

41. Morano S, Mistrangelo E, Pastorino D, Lijoi D, Costantini S, Ragni N. A randomized comparison of suturing techniques for episiotomy and laceration repair after spontaneous vaginal birth. J Minim Invasive Gynecol. 2006; 13(5):457-62. Doi: http://dx.doi.org/10.1016/j.jmig.2006.06.006

42. Elnagar WM. Median versus mediolateral episiotomy. Al-Azhar Assiut Medical Journal. 2015; 13(4):217-24. Disponible en: http://www.aamj.eg.net/journals/pdf/2712.pdf

43. Edessy M, Nasr AAM, El-Aty MGA, Ahmed W. Post Episiotomy Physical and Psychological Morbidities in Al Azhar University Hospital of Assiut -Upper Egypt. SOJ Gynecology, Obstetrics and Women's Health. 2015; 1(1):1-4. Doi: http://dx.doi.org/10.15226/2381-2915/1/1/00102

44. Sultan AH, Thakar R, Ismail KM, Kalis V, Laine K, Räisänen $\mathrm{SH}$, de Leeuw JW. The role of mediolateral episiotomy during operative vaginal delivery. Eur J
Obstet Gynecol Reprod Biol. 2019; 240:192-6. Doi: http://dx.doi.org/10.1016/j.ejogrb.2019.07.005

45. Marty N, Verspyck E. Déchirures périnéales obstétricales et épisiotomie: aspects techniques. RPC prévention et protection périnéale en obstétrique CNGOF [Perineal tears and episiotomy: Surgical procedure - CNGOF perineal prevention and protection in obstetrics guidelines]. Gynecol Obstet Fertil Senol. 2018; 46(12):948-67. Doi: http://dx.doi.org/10.1016/j.gofs.2018.10.024

46. Sagi-Dain L, Sagi S. Indications for episiotomy performance - a cross-sectional survey and review of the literature. J Obstet Gynaecol. 2016; 36(3):361-5. Doi:

http://dx.doi.org/10.3109/01443615.2015.1065233

47. Corrêa Junior $M$, Passini Júnior R. Selective episiotomy: Indications, techinique, and association with severe perineal lacerations. Rev Bras Ginecol Obstet. 2016; 38:301-7. Doi: http://dx.doi.org/10.1055/s-0036-1584942 